\title{
Optical injection method for the measurement of small linewidth enhancement factor in solid-state lasers.
}

\author{
Aurélien Thorette, Marco Romanelli, and Marc Vallet \\ Institut de Physique de Rennes, UMR Université Rennes I - CNRS 6251, Campus de Beaulieu, 35042 Rennes Cedex, France \\ aurelien.thorette@univ-rennes1.fr
}

The linewidth enhancement factor, also referred to as Henry factor or $\alpha$ factor, quantifies the intrinsic phaseamplitude coupling in a laser gain medium. Very extensive literature exists on measurement methods for semiconductor lasers, including direct estimation of the gain asymmetry, pump induced phase modulation ("AM/FM" method) and optical injection. Unfortunately none of these methods is well-suited for solid-state lasers, which feature slower dynamics and small values of $\alpha$. Few measures are available in the literature: a value of $\alpha=0.25 \pm 0.13$ was found in a Nd:YVO${ }_{4}$ laser, using injection and $\mathrm{AM} / \mathrm{FM}$ modulation methods [1], and a surprisingly large $\alpha \approx 1$ was reported in Nd:YAG microchip lasers.

Here, we propose a new "FM/AM" method based on the amplitude response of the laser to a phasemodulated injection. We show that for an injected laser, this response is minimal for a particular value $\Delta_{\mathrm{m}}$ of the frequency detuning $\Delta$ between the injected field and the laser field, and that this value is directly related to $\alpha$. Furthermore, unlike standard methods that often use pump modulation, we operate at a constant intracavity power, so the measurement is free from any thermal considerations [2]. Our method can greatly benefit of operating the laser in a dual frequency regime, that is, when the laser produces two orthogonal polarization modes with a tunable frequency difference. Indeed, this, combined with a frequency-shifted optical feedback, allow to avoid the critical need of an external stable master laser, as one mode of the laser can be used to inject the other one.

(a)

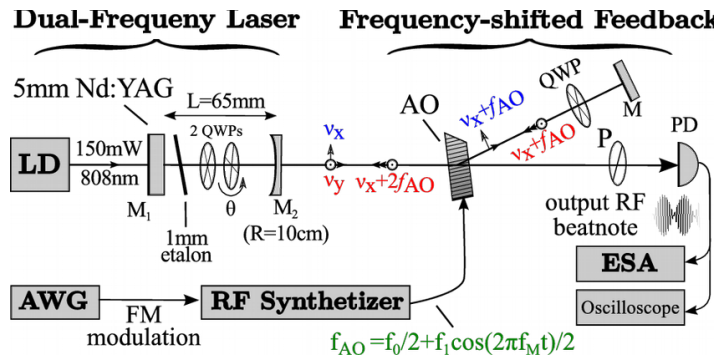

(b)

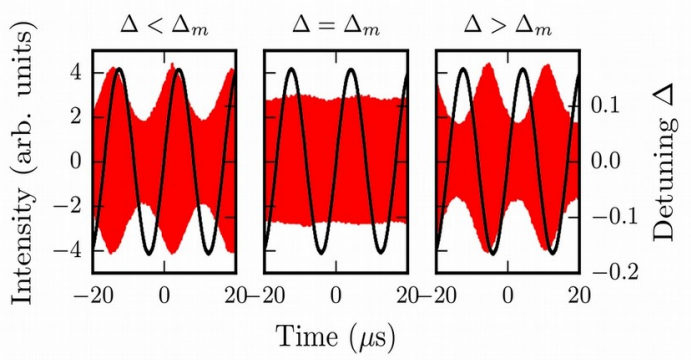

Fig. 1 (a) Experimental setup. (b) Amplitude response (red) to a phase perturbation in the form of a detuning modulation (black). A minimal amplitude response is clearly seen for a particular value of the mean detuning, and it is associated with a change in the phase sign. Based on a rate equations model, this measurement is used to deduce the value of the $\alpha$ factor in Nd:YAG.

We have implemented this measurement on a diode-pumped Nd:YAG solid-state laser (Fig. 1a), for which we have measured the "minimal response detuning" $\Delta_{\mathrm{m}}$ (Fig. 1b). Using a well-tested rate equations model allows us to deduce $\alpha$ from this measurement, taking into account the coupling between the slave and master modes inside the gain medium [3]. Performing the measurements for different injection levels has led to a rather precise characterization of a small $\alpha$ factor, which we found to be $\alpha=0.28 \pm 0.04$.

While such a small factor seem to have little influence on the laser behavior, it should be taken into account when targeting applications needing stabilized solid state lasers with very low optical phase noise. In this context, this measurement, applied to erbium-doped bulk or fibered medium, could be used to give clues on the potential contribution of $\alpha$ to the $\mathrm{AM} / \mathrm{FM}$ noise conversion process during low phase noise microwave or $\mathrm{THz}$ generation [4].

\section{References}

[1] T. Fordell, S. Valling, and Åsa Marie Lindberg, "Modulation and the linewidth enhancement factor of a diode-pumped Nd:YVO4 laser," Opt. Lett. 30, 3036 (2005).

[2] A. Thorette, M. Romanelli and M. Vallet, "Linewidth enhancement factor measurement based on FM-modulated optical injection: application to rare-earth doped active medium" Opt. Lett. (under review).

[3] M. Romanelli, L. Wang, M. Brunel and M. Vallet, "Measuring the universal synchronization properties of driven oscillators across a Hopf instability," Opt. Exp., 22, 7364 (2014).

[4] G. Pillet et al., "Dual-Frequency Laser at $1.5 \mu \mathrm{m}$ for Optical Distribution and Generation of High-Purity Microwave Signals," J. Lightwave Technol. 26, 2764 (2008). 